1 Faculty of Health Sciences and Sport, University of Stirling, Stirling, UK

2 Warrell Unit, St Mary's Hospital, Manchester University NHS Trust, Manchester Academic Health Science Centre, Manchester, UK

3 Division of Developmental Biology and Medicine, School of Medical Sciences, University of Manchester, Manchester, UK

4 Nursing, Midwifery and Allied Health Professions Research Unit, Glasgow Caledonian University, Glasgow, UK

Correspondence to C Bugge carol.bugge@stir.ac.uk Cite this as: BMJ 2021;372:n310 http://dx.doi.org/10.1136/bmi.n310 Published: 19 February 2021

UNCERTAINTIES

\title{
Does self-management of vaginal pessaries improve care for women with pelvic organ prolapse?
}

\section{Carol Bugge, ${ }^{1}$ Melanie Dembinsky, ${ }^{1}$ Rohna Kearney, ${ }^{2,3}$ Suzanne Hagen ${ }^{4}$}

\section{What you need to know}

- Self-management of vaginal pessary for pelvic organ prolapse may enable patient autonomy over when to remove and insert the pessary (for example, to remove before intercourse) and may reduce the number of clinic appointments

- Limited evidence from small observational studies suggests that self-management may improve the length of time a woman uses a pessary for, her satisfaction, and her quality of life, without increasing complication rates, but it is difficult to generalise from this evidence

- During the covid-19 pandemic, reassure women who may need a pessary change that a delay of a few months is acceptable as long as they are not experiencing any problems such as vaginal bleeding, discharge, or pain

Pelvic organ prolapse is common in women over the age of 50 and negatively affects their quality of life. ${ }^{1}$ Management includes lifestyle modification, pelvic floor muscle training, vaginal pessary, or surgery. ${ }^{2}$ Pessaries may be preferred by women as an alternative to surgery or while they await surgery. In an observational study in the UK (680 women), two thirds of women initially preferred conservative management with pessaries. Women were more likely to consider surgery if they were bothered by severe symptoms affecting bowel emptying, sexual function, and quality of life. ${ }^{3}$

A pessary is inserted into the vagina to hold up the vaginal walls and pelvic organs and to relieve symptoms of prolapse (figure 1 shows types of pessary). Two types of pessary are commonly used: support pessaries-such as rings-and space occupying pessaries-such as Gellhorn or shelf pessaries. Pessaries can be inserted by general practitioners, specialist nurses, specialist physiotherapists, or gynaecologists. Guidance from the National Institute for Health and Care Excellence (NICE) recommends removing and replacing the pessary at least every six months to prevent and detect complications such as vaginal ulceration. ${ }^{2}$ NICE guidance does not mention pessary self-management, whereas US guidelines suggest that, where possible, women should be taught to self-manage. ${ }^{4}$ Neglected pessaries can result in incarceration or the development of vesicovaginal or rectovaginal fistulae. In light of the covid-19 pandemic, the British Society of Urogynaecology recommends telephone consultations and a delayed review for women needing a pessary change and having no symptoms. ${ }^{5}$ 


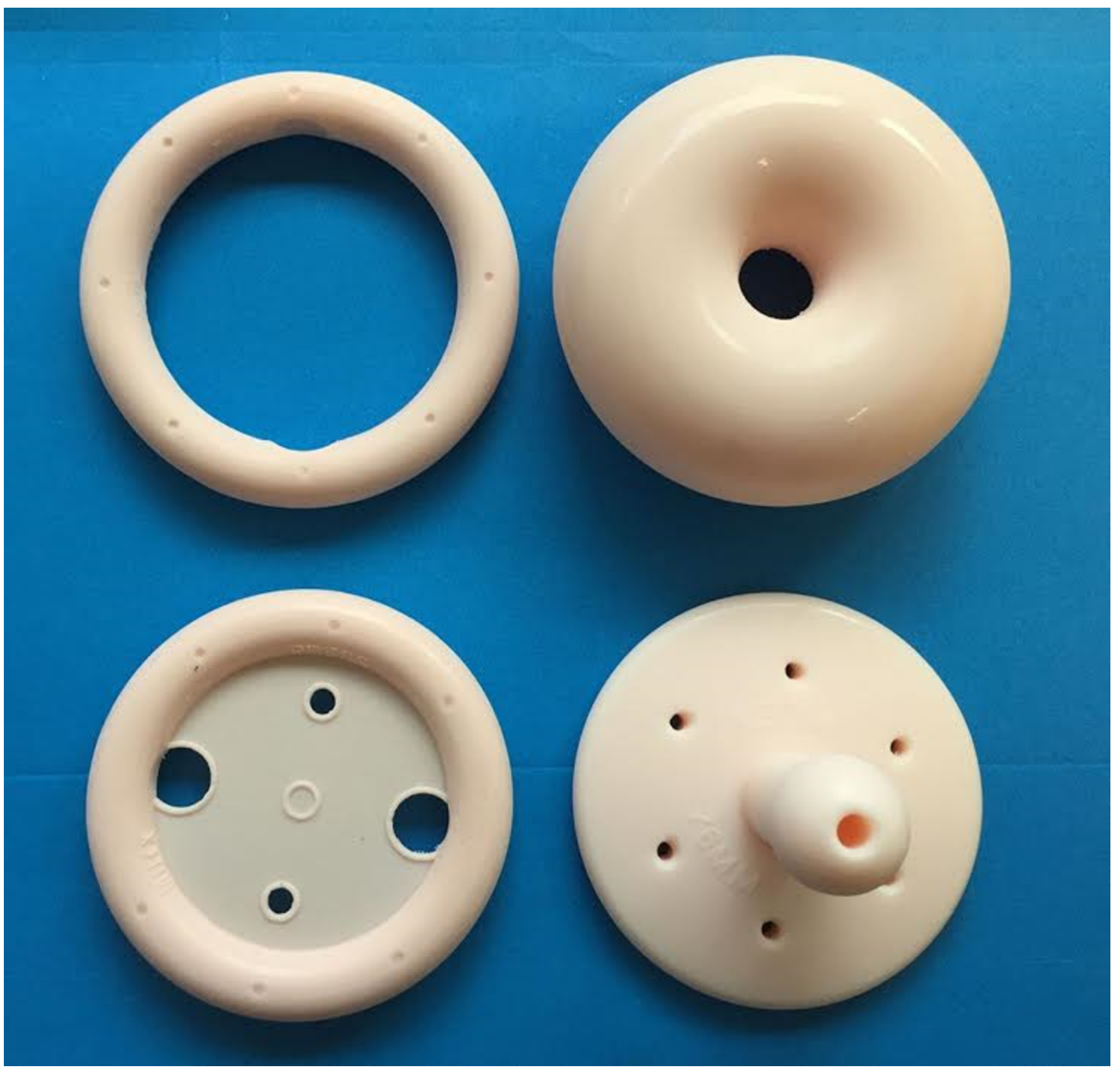

Fig 1 | Common vaginal pessaries: ring pessary (top left), doughnut (top right), ring pessary with support (bottom left), Gellhorn (bottom right)

Follow-up care can be through self-management or at a clinic. In clinic based care, women have an appointment every 4-6 months to have their pessary removed, their vaginal tissues inspected, and a new pessary inserted. ${ }^{6}$ Pessary complications such as erosions can be identified and treated early. With pessary self-management, however, a woman can remove, clean, and re-insert the pessary herself by following instructions provided by a healthcare professional. This reduces the need for clinic visits and enables flexibility on the timing of removal-for example, before having sexual intercourse. Reduced outpatient visits can have substantial cost implications for the health service. Not all practices offer pessary self-management to their patients.

It is uncertain if self-management of pessary is safe and improves quality of life for women with prolapse compared with clinic based care.

\section{What is the evidence of uncertainty?}

\section{Sources and selection criteria}

We searched the PubMed database using the terms "pessary" AND "safety" AND "self-management” AND “prolapse” on 10 August 2019 and 17 January 2021.

Evidence on pessary self-management is limited and from small non-randomised studies ${ }^{7-11}$ (table 1 , supplementary file). The studies mainly report on the length of time for which women continue to use a pessary. Overall, self-management may improve pessary continuation rates and enhance patient satisfaction and quality of life, but it is difficult to generalise these findings. The studies were conducted in different populations, were small, and lacked randomised comparison groups. ${ }^{7-11}$ Uncertainty exists over optimal 
follow-up intervals, if they are required, for women who are self-managing. One cohort study did not find an increase in complications with self-management when follow-up intervals were increased to nine months. ${ }^{11}$

\section{Is ongoing research likely to provide relevant evidence?}

We searched the US National Library of Medicine database and ISRCTN and ANZCTR registries most recently on 8 February 2021 for ongoing trials on pelvic organ prolapse. We identified two trials on pessary self-management. These trials are expected to provide some evidence on the safety and acceptability of self-management, but do not address uncertainties such as optimal follow-up times, potential differences in managing pessaries made of different materials, and patient acceptability and satisfaction.

The TOPSY study (ISRCTN62510577) ${ }^{12}$ is an ongoing randomised controlled trial in the UK that compares clinic based care with a self-management intervention for women with any type and stage of prolapse using various pessary types (340 participants recruited; expected to report in February 2022). The primary outcome measure is prolapse specific quality of life at 18 months. The study will collect data on complications and adverse events, and will include an economic evaluation. ${ }^{12}$ The second study (ACTRN12618000416291) compares self-management for PVC ring pessary with a silicon irregular hexagon pessary. The primary outcome is retention of the pessary at six and 12 months post-randomisation.

\section{What should we do in light of the uncertainty?}

The stage of prolapse, impact on quality of life, and patient preferences and characteristics guide decisions on management. In women considering pessary use, discuss options of self-management and clinic based care. Explain the importance of pessary removal and re-insertion to reduce the risk of complications. Self-management is unsuitable for women with cognitive impairment or a physical difficulty, ${ }^{2}$ such as those who do not have the manual dexterity required for pessary handling. If a woman wishes to self-manage her pessary, provide clear instructions (box 1).

\section{Box 1: What to discuss with women before pessary use ${ }^{2}$}

- Discuss all options for managing symptoms, including lifestyle modifications, pelvic floor muscle training, vaginal pessary, and surgery

- Explain that pessary use requires regular pessary changes, at least once every six months

- Self-management has the potential benefits of being able to remove and insert the pessary when desired-for example, before intercourse-and may also mean reduced clinic appointments

- If the woman prefers self-management of pessary, provide instructions on

- removal and re-insertion technique, ensuring she understands the pelvic anatomy

cleaning of the pessary, with a short explanation of the material of the pessary

- how to receive a replacement pessary

- how she should access support if she experiences problems, such as a phone number to call

- Ensure the type of pessary is appropriate for sexual activity if the woman wishes to be sexually active. Vaginal intercourse is possible with a ring pessary in place but not possible with other types of pessary in place. Self-management of space occupying pessaries tends to be more difficult than for ring and sieve pessaries
- Explain that it may take more than one fitting to achieve a successful pessary fit. The woman may need to try a pessary to find one that is most suitable. Ensure women can void after first fitting before leaving the clinical area.

- Common complications include vaginal discharge, ulceration, bleeding, and expulsion. These can occur if a pessary is not changed regularly. Less commonly, incarceration can occur, which requires pessary removal under anaesthetic. Other complications include rectovaginal or vesicovaginal fistula, and very rarely vaginal, squamous cell cancer.

Evidence for length of time between follow-up visits for clinic based care is uncertain, ${ }^{13}{ }^{14}$ with six monthly visits being common in the UK. ${ }^{6}$ No evidence based timeframe exists for a clinician's review if a woman is self-managing and not experiencing problems. A follow-up appointment every two years is reasonable in the absence of further guidelines. As the woman is not seen in clinic regularly, it is possible that complications may be missed. Provide a telephone number to call if they experience any problems, so they can be seen in clinic for a visual examination. Encourage the woman to seek medical care if she experiences excessive vaginal discharge or bleeding, or pessary dislodgement or expulsion.

\section{Recommendation for further research}

These recommendations are informed by evidence reviewed in the 6th International Consultation on Incontinence ${ }^{15}$ and a recent James Lind Alliance Priority Setting. ${ }^{16}$

Large, multicentre trials are needed to examine:

- Long term follow-up of women who self-manage to assess pessary continuation rates linked to women's quality of life and safety

- Optimal management protocols for pessary self-management (eg, follow-up timelines). Acceptability and complications with different pessary types and materials with self-management

- Clinical trials comparing self-management of vaginal pessaries between women with different stages of prolapse to assess effectiveness, safety, and quality of life

\section{What patients need to know}

- If you have a pelvic organ prolapse, your doctor may suggest inserting a vaginal pessary to reduce symptoms

- It may take more than one appointment to find a suitable pessary and for appropriate fitting

- A pessary must be removed and cleaned or replaced at least once every six months

- You can have regular pessary appointments for removal and re-insertion, or learn to self-manage with access to support when required

- No evidence exists to guide whether self-management or clinic based care is better in terms of reduced complications or improved quality of life and patient acceptability. Discuss your preferences with your doctor and decide a suitable follow-up approach

- Seek medical advice if the pessary is uncomfortable or if you experience non-menstrual vaginal bleeding, excessive vaginal discharge, or urinary or bowel problems

\section{Education into practice}

- How would you discuss the options of self-management and clinic based management with a woman considering pessary use for prolapse? 
- What guidance would you provide to a woman opting for self-management of vaginal pessary?

\section{How patients were involved in this article}

A patient reviewed this manuscript. She highlighted the importance of having telephone support available when self-managing a pessary. This would help ease her concerns in the time between vaginal examinations every two years. Telephone support as a component of self-management has been suggested in the manuscript. We are grateful for the patient's input.

Competing interests: We have read and understood The BM/ policy on declarations of interest. CB, $\mathrm{SH}$, and $\mathrm{MD}$ declare that there are none. RK was the topic lead for prolapse on the NICE guidelines (National, Guideline Alliance UK. Urinary incontinence and pelvic organ prolapse in women: management. 2019).

Contributorship statement and guarantor: $\mathrm{CB}$ acts as guarantor and takes overall responsibility for the content of the paper; RK is responsible for the clinical content. MD and RK initially drafted the manuscript. CB, SH and RK conceptualised and developed the TOPSY study. All authors contributed substantially to the paper content, and reviewed and edited the manuscript.

Funding statement: The TOPSY Trial is funded by the National Institute for Health Research (NIHR) (Health Technology Assessment Programme (project reference 16/82/01)). The views expressed are those of the author(s) and not necessarily those of the NIHR or the Department of Health and Social Care.

Provenance and peer review: commissioned, based on an idea from the authors; externally peer reviewed.

1 Hendrix SL, Clark A, Nygaard I, Aragaki A, Barnabei V, McTiernan A. Pelvic organ prolapse in the Women's Health Initiative: gravity and gravidity. Am J Obstet Gynecol 2002;186:1160-6. doi: 10.1067/mob.2002.123819 pmid: 12066091

2 National Institute for Health and Care Excellence. Urinary incontinence and pelvic organ prolapse in women: management. 2019. https://www. nice.org.uk/guidance/ng123

3 Kapoor DS, Thakar R, Sultan AH, Oliver R. Conservative versus surgical management of prolapse: what dictates patient choice?Int Urogynecol J Pelvic Floor Dysfunct 2009;20:1157-61.

4 American College of Obstetricians and Gynecologists. Pelvic organ prolapse. ACOG Practice Bulletin. Obstetr Gynecol 2019. https://journals. Iww.com/greenjournal/Abstract/2019/11000/Pelvic_Organ_Prolapse_ACOG_Practice_Bulletin.44.aspx

5 Royal College of Obstetricians and Gynaecologists. BSUG Guidance on management of urogynaecological conditions and vaginal pessary use during the Covid 19 pandemic. https://www.rcog.org.uk/globalassets/documents/guidelines/2020-04-09-bsug-guidance-onmanagement-of-urogynaecological-conditions-and-vaginal-pessary-use-during-the-covid-19pandemic.pdf

6 Bugge C, Hagen S, Thakar R. Vaginal pessaries for pelvic organ prolapse and urinary incontinence: a multi-professional survey of practice. Int Urogynecol / 2013;24:1017-24.

7 Kearney R, Brown C. Self-management of vaginal pessaries for pelvic organ prolapse. BMJ Qual Improv Rep 2014;3:4206180.w2533.

8 Nemeth Z, Nagy S, Ott J. The cube pessary: an underestimated treatment option for pelvic organ prolapse? Subjective 1-year outcomes. Int Urogynecol J 2013;24:1695-701. doi: 10.1007/s00192-013-2093-z pmid: 23579291

9 Manonai J, Sarit-Apirak S, Udomsubpayakul U. Vaginal ring pessary use for pelvic organ prolapse: continuation rates and predictors of continued use. Menopause 2019;26:665-9. doi: 10.1097/GME.0000000000001277 pmid: 30562318

10 Chien C-W, Lo T-S, Tseng L-H, Lin Y-H, Hsieh W-C, Lee S-J. Long-term outcomes of self-management Gellhorn pessary for symptomatic pelvic organ prolapse. Female Pelvic Med Reconstr Surg 2020;26:e47-53.pmid: 31393340

11 Thys SD, Hakvoort RA, Asseler J, Milani AL, Vollebregt A, Roovers JP. Effect of pessary cleaning and optimal time interval for follow-up: a prospective cohort study. Int Urogynecol J Pelvic Floor Dysfunc 2020;31:1567-74.

12 Hagen S, Kearney R, Goodman K, etal. Clinical and cost-effectiveness of vaginal pessary self-management compared to clinic-based care for pelvic organ prolapse: protocol for the TOPSY randomised controlled trial. Trials 2020;21:837. doi: 10.1186/s13063-020-04738-9 pmid: 33032644

13 Tam MS, Lee VYT, Yu ELM, etal. The effect of time interval of vaginal ring pessary replacement for pelvic organ prolapse on complications and patient satisfaction: A randomised controlled trial. Maturitas 2019;128:29-35. do: 10.1016/..maturitas.2019.07.002 pmid: 31561819

14 Propst K, Mellen C, O'Sullivan DM, Tulikangas PK. Timing of office-based pessary care: a randomized controlled trial. Obstet Gynecol 2020;135:100-5.

15 Dumoulin C, Adewuyi T, Bradley C, etal. Adult conservative management. In: Abrams P., Cardozo L. Wagg A, Wein AJ, eds. Incontinence: 6th International Consultation on Incontinence, Tokyo. England International Continence Society, 2017: 1443-628.
16 Lough K, Hagen S, McClurg D, Pollock AJLA Pessary PSP Steering Group. Shared research priorities for pessary use in women with prolapse: results from a James Lind Alliance Priority Setting Partnership. BMJ Open 2018;8:e021276. doi: 10.1136/bmjopen-2017-021276 pmid: 29705767 